\title{
Protection of the Marginal Mandibular Branches of the Facial Nerves by Different Surgical Procedures in Comprehensive Cervical Lymphadenectomy for Locally Advanced Oral and Oropharyngeal Cancer: A Multicenter Experience
}

Jiuzhou Zhao

Longgang E.N.T hospital \& Shenzhen Key Laboratory of E.N.T, Institute of E.N.T Shenzhen, Guangdong Province

\section{Weng Zen}

Tumor Hospital of Ganzhou

\section{Chengyu Qiu}

First Hospital of Qiqihaer city

Jiafeng Liu

First Affiliated Hospital of Gannan Medical University

Ke Li

Longgang E.N.T hospital \& Shenzhen Key Laboratory of E.N.T, Institute of E.N.T Shenzhen, Guangdong Province

Michael C. F. Tong

The Chinese University of Hong Kong

Xiangmin Zhang ( $\triangle$ Xmzhang019@126.com)

Longgang E.N.T hospital \& Shenzhen Key Laboratory of E.N.T, Institute of E.N.T Shenzhen, Guangdong Province

\section{Research Article}

Keywords: Head and neck surgery, cervical lymphadenectomy, marginal mandibular branches of facial nerves, surgical approach

Posted Date: January 24th, 2022

DOI: https://doi.org/10.21203/rs.3.rs-1271722/v1

License: (c) (i) This work is licensed under a Creative Commons Attribution 4.0 International License. Read Full License 


\section{Abstract}

Background and objective: Tumor treatment has been progressive. Targeted therapy and immunotherapy have contributed in the surgical success of several patients with advanced oral and oropharyngeal cancer. Surgery is the primary treatment for oral and oropharyngeal cancer, and cervical lymphadenectomy is crucial in surgery. Evidence has shown that submandibular glands can be preserved in cervical lymphadenectomy for early stage oral and oropharyngeal cancer; however, the removal of the submandibular glands is inevitable in cervical lymphadenectomy for locally advanced oral and oropharyngeal cancer. Nowadays, with individualized and precise treatment, the anatomy and protection of the marginal mandibular branches of the facial nerves have received increasing attention. In this study, according to the different characteristics of patients and cervical lymph node metastasis of oral and oropharyngeal cancer, the marginal mandibular branches of facial nerves were treated by different surgical procedures, and the safety and protective effects of different surgical procedures were investigated.

Methods: From January 2014 to June 2021, 197 patients with oral and oropharyngeal cancer satisfying the inclusion criteria were selected from the Head and Neck Department of Shenzhen Otolaryngology Research Institute/Shenzhen Longgang Otolaryngology Hospital, Head and Neck Department of Gannan Medical University Affiliated Cancer Hospital, Department of Oral and Maxillofacial Surgery of the First Hospital of Qiqihar in Heilongjiang Province, and Department of Otorhinolaryngology-Head and Neck Surgery of First Affiliated Hospital of Gannan Medical University. All patients underwent radical surgery by comprehensive cervical lymphadenectomy or radical surgery plus repair and reconstruction. During the operation, the marginal mandibular branches of the facial nerves were treated and the submandibular glands were removed. According to the different characteristics of patients and cervical metastatic lymph nodes, three different surgical procedures were used to treat the marginal mandibular branches of the facial nerve: finding and exposing the marginal mandibular branches of the facial nerves at the mandibular angles of the platysma flaps, finding and exposing the marginal mandibular branches of facial nerves at the intersections of the distal ends of facial arteries and veins with the mandible, and not exposing the marginal mandibular branches of the facial nerves. The anatomical position, injury, and complications of the marginal mandibular branches of the facial nerves were observed.

Results: The marginal mandibular branches of the facial nerves penetrated from the front or lower ends of the parotid glands. They were constantly located between the deep surface of the platysma and the superficial layer of the deep fascia cervicalis, and on the plane of the lower mandible. They crossed the superficial surface of the posterior facial veins, mandibular angles, and anterior facial veins from back to front and entered the deep surface of deltoid muscles at the distal ends of facial arteries and veins. Among the 197 oral and oropharyngeal cancer patients undergoing comprehensive cervical lymphadenectomy with excision of the submandibular glands, the marginal mandibular branches of the facial nerves were found and exposed at the mandibular angles of the platysma flaps in 102 patients (102/197, 51.78\%); the marginal mandibular branches of facial nerves were found and exposed at the intersections of the distal ends of the facial arteries and veins with the mandibles in 64 patients $(64 / 197$, 
$32.49 \%)$; the marginal mandibular branches of facial nerves were not exposed in 31 patients (31/197, $15.73 \%$ ); the two methods for finding the marginal mandibular branches of the facial nerves both successfully found the marginal mandibular branches. Among the 197 patients with oral and oropharyngeal cancer, four patients $(4 / 197,2.03 \%)$ had permanent injury of the marginal mandibular branches of the facial nerves. Among them, one patient $(1 / 102,0.98 \%)$ had injury of the marginal mandibular branches of the facial nerves found and exposed at the mandibular angles of platysma flaps; one patient $(1 / 64,1.56 \%)$ had injury of the marginal mandibular branches of the facial nerves found and exposed at the intersections of the distal ends of the facial arteries and veins with the mandible; the marginal mandibular branches of the facial nerves were not found and exposed in two patients $(2 / 31$, $6.45 \%)$, and the differences were not statistically significant $(P>0.05)$. Temporary injury occurred in seven patients $(7 / 197,3.55 \%)$. Among them, two patients $(2 / 102,1.96 \%)$ had injury of the marginal mandibular branches of the facial nerves found and exposed at the mandibular angles of platysma flaps; two patients $(2 / 64,3.13 \%)$ had injury of the marginal mandibular branches of the facial nerves found and exposed at the intersections of the distal ends of facial arteries and veins with the mandible; and the marginal mandibular branches of facial nerves were not found and exposed in three patients $(3 / 31$, $9.68 \%)$. The differences were not statistically significant $(P>0.05)$. The patients were followed up for 6 to 90 months after surgery, and the functions were restored in all patients with temporary injury of the marginal mandibular branches of the facial nerves.

Conclusion: The three different surgical procedures were all safe and effective in treating the marginal mandibular branches of the facial nerves. Finding and exposing the marginal mandibular branches of the facial nerves at the mandibular angles of the platysma flaps was suitable for those with larger and more lymph nodes in region $\mathrm{lb}$. Finding and exposing the marginal mandibular branches of the facial nerves at the intersections of the distal ends of facial arteries and veins with the mandible was suitable for those with larger and more lymph nodes in region lla. Not exposing the marginal mandibular branches of the facial nerves was suitable for those without obvious lymph nodes in region lb or region lla. Intraoperatively, we should choose an appropriate method in dealing with the marginal mandibular branches of the facial nerves according to the different characteristics of patients and cervical metastatic lymph nodes as well as the proficiency of surgeons.

\section{Introduction}

Comprehensive cervical lymphadenectomy includes all the lymphoid and adipose tissues that can be dissected in classical radical cervical lymphadenectomy, and whether the internal jugular veins, sternocleidomastoid muscles, and accessory nerves are preserved does not affect whether it is classified as comprehensive [1]. Radical resection or radical resection plus repair and reconstruction with comprehensive cervical lymphadenectomy is the main method for comprehensive treatment of locally advanced oral and oropharyngeal cancer, although there is evidence that the submandibular glands can be spared in cervical lymphadenectomy for early stage oral and oropharyngeal cancer [2-5]. However, removal of the submandibular glands is inevitable in cervical lymphadenectomy for locally advanced oral and oropharyngeal cancer [6-8]. 
Surgeries involving the facial nerves have a high risk of iatrogenic injuries. The marginal mandibular branches of the facial nerves are extremely special in nature. Once an injury occurs, the complications will be devastating and functions will be lost, which will involve the patient's facial aesthetics [9-11], causing great psychological trauma to the patient, and thus affecting tumor treatment $[12,13]$. Whether it is necessary to dissect the marginal mandibular branches of the facial nerves when removing the submandibular glands during comprehensive cervical lymphadenectomy, and whether the dissection can protect the marginal mandibular branches of the facial nerves are a subject worthy of clinical research. In this study, according to the different characteristics of patients and cervical lymph node metastasis of oral and oropharyngeal cancer, different surgical procedures were used to treat the marginal mandibular branches of the facial nerves, and the safety and protective effects of different surgical procedures were investigated, so as to further summarize the clinical experience, reduce the risk of iatrogenic injury to the marginal mandibular branches of the facial nerves, and maintain the aesthetics and functions of patients' faces to the maximum extent.

\section{Materials And Methods}

\section{Ethics statement}

The Ethical Committee of the Longgang E.N.T hospital approved clinical samples for research purposes (NO. 2022-0001), and this study was confirmed to the principles contained in the World Medical Association Declaration of Helsinki. Informed consent was requested as anonymous specimens and was given by all human participants in this study.

\section{Case inclusion and exclusion criteria}

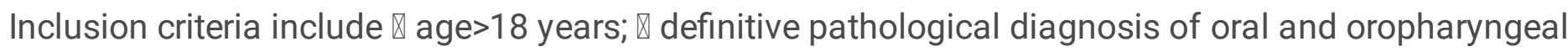
cancer requiring cervical lymphadenectomy with submandibular gland resection according to the National Comprehensive Cancer Network guidelines or the results of multidisciplinary care discussion; $\mathbb{\bigotimes}$ no significant invasion of the marginal mandibular branches of the facial nerves before operation; $\nabla$ no serious cardiovascular diseases, diabetes, chronic respiratory diseases, cerebrovascular diseases, etc.; \Karnofsky score $\geq 80$ points; $\otimes$ an estimated survival of more than one year; $\otimes$ and patients who voluntarily participated and signed the informed consent form.

Exclusion criteria include $₫$ cervical lymphadenectomy for oral and oropharyngeal cancer without the need for submandibular gland resection; $₫$ significant invasion of the marginal mandibular branches of the facial nerves before operation; $\otimes$ significant submandibular lesions; $\nabla$ a history of surgery or radiotherapy of the neck; $\Downarrow$ distant metastasis; $\varangle$ unable to tolerate the surgery due to severe comorbidities.

\section{Clinical data}


From January 2014 to June 2021, 197 patients with oral and oropharyngeal cancer, including 131 males and 66 females, aged 29 to 68 years, and with a median age of 54.6 years, were selected from the Head and Neck Department of Shenzhen Otolaryngology Research Institute/Shenzhen Longgang Otolaryngology Hospital, Head and Neck Department of Gannan Medical University Affiliated Cancer Hospital, Department of Oral and Maxillofacial Surgery of the First Hospital of Qiqihar in Heilongjiang Province, and Department of Otorhinolaryngology-Head and Neck Surgery of First Affiliated Hospital of Gannan Medical University. There were 78 cases of tongue cancer, 35 cases of gingival cancer, 32 cases of buccal cancer, 28 cases of oral floor cancer, 24 cases of lingual root or oropharyngeal cancer, 16 cases at stage III, 114 cases at stage IVA, and 67 cases at stage IVB. All patients underwent radical tumor resection or radical tumor resection plus flap repair and reconstruction with cervical lymphadenectomy and submandibular gland resection. The marginal mandibular branches of the facial nerves were treated and submandibular glands were removed during the operation (Figs 1-6).

\section{Evaluation criteria for facial nerve function}

The facial nerve function was evaluated using the Sunnybrook [14] facial nerve evaluation system. According to this scoring criteria, the higher the score, the better the facial nerve function. The facial nerve function is normal if the scores before and after surgery are the same, and the score after surgery being lower than that before surgery indicates facial nerve dysfunction. In this study, the function of the marginal mandibular branches of the facial nerves was mainly evaluated.

\section{Surgical approaches}

All operations were performed by doctors with the title of associate chief physician or above and with several years of surgical experience. The most suitable surgical incision was selected according to the characteristics of oral and oropharyngeal cancer and the results of the multidisciplinary care discussion. According to the different characteristics of patients and cervical metastatic lymph nodes, three different surgical procedures were used to treat the marginal mandibular branches of the facial nerves: finding and exposing the marginal mandibular branches of the facial nerves at the mandibular angles of the platysma flaps, finding and exposing the marginal mandibular branches of the facial nerves at the intersections of the distal ends of facial arteries and veins with the mandible, and not exposing the marginal mandibular branches of the facial nerves.

Finding and exposing the marginal mandibular branches of the facial nerves at the mandibular angles of the platysma flaps: At the junction of about $1 \mathrm{~cm}$ superior to the mandibular angle and $1 \mathrm{~cm}$ anterior to the ascending branch of the mandible, an electric scalpel was used to make an incision along the horizontal plane of the mandible, and then the adipose tissues were carefully cut open, the marginal mandibular branch of the facial nerves were located up and down, and the mandibular marginal branch of the facial nerve was separated during the whole process. Then, the distal ends of the facial artery and vein were separated and disconnected along the surface of the submandibular gland, and the submandibular gland and the lymphoid adipose in region lb were separated and resected (Fig 7). Finding and exposing the marginal mandibular branches of the facial nerves at the intersections of the distal 
ends of the facial arteries and veins with the mandible: After the platysma flap was overturned, the distal ends of the facial artery and vein were found, and careful separation was conducted. The marginal mandibular branch of the facial nerve was found on the superficial surface of the facial artery and vein. The marginal mandibular branch of facial nerve was dissected throughout by two-way separation. The distal ends of the facial artery and vein were disconnected. The submandibular gland as well as the lymphoid and adipose tissues in region lb were separated and excised (Fig 8). Not exposing the marginal mandibular branches of the facial nerves: The superficial layer of the deep cervical fascia was used as the anatomical plane, and the surrounding tissues were carefully separated along the gland surface. During the process, the gland was slightly pulled down with devices to maintain a safe distance from the marginal mandibular branch and the submandibular gland body; moreover, the distal ends of the facial artery and vein were exposed, the facial artery and vein were disconnected close to the surface of the submandibular gland, blunt dissection was conducted along the circumference of the submandibular gland, and the lymphoid and adipose tissues in region lb were separated and excised.

\section{Selection of surgical procedures to protect the marginal mandibular branches of the facial nerves}

The three different surgical procedures were all safe and effective in treating the marginal mandibular branches of the facial nerves. Finding and exposing the marginal mandibular branches of the facial nerves at the mandibular angles of the platysma flaps was suitable for those with larger and more lymph nodes in region $\mathrm{Ib}$. Finding and exposing the marginal mandibular branches of the facial nerves at the intersections of the distal ends of facial arteries and veins with the mandible was suitable for those with larger and more lymph nodes in region lla. Not exposing the marginal mandibular branches of the facial nerves was suitable for those without obvious lymph nodes in region Ib or region Ila.

\section{Postoperative observation items}

The anatomical position and state of the marginal mandibular branches of the facial nerves, as well as the function of the marginal mandibular branches of the facial nerves after surgery, were observed.

\section{Statistical methods}

The anatomical state and injury, as well as occurrence and recovery of complications of the marginal mandibular branches of facial nerves under the three different surgical procedures, were statistically analyzed. Chi-square test was conducted using SPSS 20.0, and the difference was considered statistically significant when $P<0.05$.

\section{Results}

\section{Anatomical position of the marginal mandibular branches of the facial nerves}

The marginal mandibular branches of the facial nerves penetrated from the front or lower ends of the parotid glands. They were constantly located between the deep surface of the platysma and the superficial layer of the deep fascia cervicalis, and on the plane of the lower mandible. They crossed the 
superficial surface of the posterior facial veins, mandibular angles, and anterior facial veins from back to front, and entered the deep surface of deltoid muscles at the distal ends of facial arteries and veins.

\section{Different surgical procedures to deal with the marginal mandibular branches of the facial nerves}

Among the 197 oral and oropharyngeal cancer patients undergoing comprehensive cervical lymphadenectomy with excision of the submandibular glands, the marginal mandibular branches of the facial nerves were found and exposed at the mandibular angles of the platysma flaps in 102 patients $(102 / 197,51.78 \%)$; the marginal mandibular branches of the facial nerves were found and exposed at the intersections of the distal ends of facial arteries and veins with the mandibles in 64 patients $(64 / 197$, $32.49 \%)$; the marginal mandibular branches of the facial nerves were not exposed in 31 patients (31/197, $15.73 \%$ ); the two methods for finding the marginal mandibular branches of the facial nerves both successfully found the marginal mandibular branches.

\section{Injury of the marginal mandibular branches of the facial nerve}

Among the 197 patients with oral and oropharyngeal cancer, four patients $(4 / 197,2.03 \%)$ had permanent injury of the marginal mandibular branches of the facial nerves. Among them, one patient $(1 / 102,0.98 \%)$ had injury of the marginal mandibular branches of the facial nerves found and exposed at the mandibular angles of the platysma flaps; one patient $(1 / 64,1.56 \%)$ had injury of the marginal mandibular branches of the facial nerves found and exposed at the intersections of the distal ends of the facial arteries and veins with the mandible; the marginal mandibular branches of facial nerves were not found and exposed in two patients $(2 / 31,6.45 \%)$; the differences were not statistically significant $(P>0.05$; $\chi^{2}=3.6820, P=0.1587$ ).

Temporary injury occurred in seven patients (7/197, 3.55\%). Among them, two patients $(2 / 102,1.96 \%)$ had injury of the marginal mandibular branches of the facial nerves found and exposed the mandibular angles of the platysma flaps; two patients $(2 / 64,3.13 \%)$ had injury of the marginal mandibular branches of the facial nerves found and exposed at the intersections of the distal ends of the facial arteries and veins with the mandible; and the marginal mandibular branches of the facial nerves were not found and exposed in three patients $(3 / 31,9.68 \%)$. The differences were not statistically significant $(P>0.05)$. The patients were followed up for 6-90 months after surgery, and the functions were restored in all patients with temporary injury of the marginal mandibular branches of the facial nerves.

\section{Discussion}

Cervical lymph node metastasis is a characteristic of locally advanced oral and oropharyngeal cancer and an important factor for prognosis. Good control of lymph nodes is extremely important for improving the survival rate and quality of life in locally advanced oral and oropharyngeal cancer $[15,16]$. Lymphadenectomy is the gold standard for surgical treatment of cervical lymph node metastasis. With the development of functional surgery, radical cervical lymphadenectomy characterized by the resection of internal jugular veins, sternocleidomastoid muscles, and accessory nerves has been used decreasingly 
$[17,18]$. Under the concept of maximum dissection of cervical lymphoid and adipose tissues and maximum preservation of normal tissues, whether the internal jugular veins, sternocleidomastoid muscles, accessory nerves, and other tissue structures are spared, does not affect the thoroughness of cervical lymphadenectomy, and selective lymphadenectomy and comprehensive lymphadenectomy are more in line with the concept of functional surgery [1]. Under the guidance of the concept of functional surgery, the preservation of submandibular glands in cervical lymphadenectomy has received increasing attention. There is enough evidence showing that it is safe and feasible to preserve the submandibular glands in cervical lymphadenectomy for early stage oral and oropharyngeal cancer; however, it is still necessary to remove the submandibular glands in cervical lymphadenectomy for locally advanced oral and oropharyngeal cancer [19-21], and the removal of submandibular glands is closely related to marginal mandibular branches of the facial nerves [22,23].

The anatomy of the marginal mandibular branches of the facial nerves are complicated. The facial nerves run forward and downward from the stylomastoid foramina, pass through the posteromedial surface of parotid glands, and enter the parotid glands. The marginal mandibular branches are sent out from the cervical-facial trunks, pass through the front or lower end of parotid glands, pass through the mandibular angles, and are constantly located between the deep surface of platysma and the superficial layer of deep fascia cervicalis, and on the plane of the lower mandible. They cross the superficial surface of the posterior facial veins, mandibular angles, and anterior facial veins from back to front and enter the deep surface of the deltoid muscles at the distal ends of facial arteries and veins [24-26]. A marginal mandibular branch of the facial nerve is a fine nerve, which usually has 1-3 branches. After its injury, it leads to paralysis of the lower lip, bilateral facial asymmetry, significantly skewed mouth angle, salivation, and even impact on eating, which seriously affects the appearance and functions of patients, reduces the quality of life, and causes great psychological trauma to patients $[9,27]$. How to protect the marginal mandibular branches of the facial nerves when resecting the submandibular glands in comprehensive cervical lymphadenectomy in patients with locally advanced oral and oropharyngeal cancer, whether to dissect them and how to dissect, are worth pondering.

In our multicenter retrospective study, according to the different characteristics of patients and cervical metastatic lymph nodes, as well as the proficiency of surgeons, we adopted three different surgical procedures to deal with the marginal mandibular branches of the facial nerves. For patients with larger and more lymph nodes in region $\mathrm{lb}$, the marginal mandibular branches of the facial nerves were found and exposed at the mandibular angles of the platysma flaps. For patients with larger and more lymph nodes in region lla, the marginal mandibular branches of the facial nerves were found and exposed at the intersections of the distal ends of facial arteries and veins with the mandible. After finding the marginal mandibular branches of facial nerves in these two ways, the facial nerves were completely separated under direct vision, the proximal and distal ends of facial arteries and veins were disconnected, the submandibular glands were resected, and the lymphoid and adipose tissues in region Ib were cleaned. The dissection of the marginal mandibular branches of the facial nerves according to different characteristics of lymph nodes can avoid the inflammation and tissue adhesion caused by lymph nodes, and protect marginal mandibular branches of facial nerves to the greatest extent. For patients without 
obvious lymph nodes in region Ib or region Ila, the submandibular glands were removed, and the lymphoid and adipose tissues in region lb were cleaned without exposing the marginal mandibular branches of the facial nerves. The three different surgical procedures were safe, without a significant difference in causing permanent or temporary injury of the marginal mandibular branches of the facial nerves, and the protective effect was optimal.

Among the 197 patients with locally advanced oral and oropharyngeal cancer, the incidence of permanent injury of the marginal mandibular branches of the facial nerves was $2.03 \%$, and that of temporary injury was $3.55 \%$. The extremely low incidence of injury of the marginal mandibular branches of the facial nerves verified our correct choices. We should conduct sufficient evaluation before operation, formulate a strict surgical plan, and flexibly choose the measures to protect the marginal mandibular branches of the facial nerves according to the specific situation of the primary lesion and lymph nodes as well as the surgeon's proficiency. When the marginal mandibular branches of the sectional nerves are not dissected, the glands should be pulled down slightly with instruments to keep a safe distance from the marginal mandibular branches for resection. When dissecting the marginal mandibular branches of the facial nerves, we should pay attention to its branches $[25,26]$. With thorough hemostasis, the nerves can be clearly and completely exposed during dissection. Electrocoagulation and clamping are prohibited in the peripheral areas of the nerves. The epineuria of nerves should be protected, and exposure of nerves should be avoided. Various risk factors that can cause injury to the marginal mandibular branches of the facial nerves should be minimized, thereby achieving the goal of maximum protection of the marginal mandibular branches of the facial nerves.

\section{Conclusion}

With the development and progress of society, in the treatment of patients with locally advanced oral and oropharyngeal cancer, not only the survival rate but also the quality of life should be improved. The injury of the marginal mandibular branches of the facial nerves can cause dysfunctions, with serious physiological and psychological impact on patients. In the comprehensive treatment of locally advanced oral and oropharyngeal cancer, individualized and precise treatment is required, and every detail in the treatment should be finely managed. Protecting the marginal mandibular branches of the facial nerves from injury will play an increasingly important role in the comprehensive treatment of locally advanced oral and oropharyngeal cancer.

The three different surgical procedures were all safe and effective in treating the marginal mandibular branches of the facial nerves. Finding and exposing the marginal mandibular branches of the facial nerves at the mandibular angles of the platysma flaps was suitable for those with larger and more lymph nodes in region $\mathrm{Ib}$. Finding and exposing the marginal mandibular branches of the facial nerves at the intersections of the distal ends of facial arteries and veins with the mandible was suitable for those with larger and more lymph nodes in region Ila. Not exposing the marginal mandibular branches of the facial nerves was suitable for those without obvious lymph nodes in region Ib or region Ila. Intraoperatively, we should choose an appropriate method in dealing with the marginal mandibular branches of the facial 
nerves according to the different characteristics of patients and cervical metastatic lymph nodes as well as the proficiency of surgeons.

\section{Declarations}

\section{Acknowledgements}

None

\section{Authors' contributions}

JZ completed most of the experiments, and WZ, CQ, JL, CFT and $\mathrm{KL}$ assisted in completing the experiments. WZ, CQ, JL, and $\mathrm{KL}$ completed data collection and statistics. $\mathrm{XZ}$ designed and supervised the completion of this experiment and wrote this article. The authors read and approved the final manuscript.

\section{Funding}

Shenzhen Key Medical Discipline Construction Fund (No. SZXK039).

\section{Availability of data and materials}

Datasets are available on request from the corresponding author on reasonable request. The raw data and all related documents supporting the conclusions of this manuscript will be made available by the authors, without undue reservation, to any qualified researcher.

\section{Ethics approval and consent to participate}

This retrospective chart review study involving human participants was in accordance with the ethical standards of the institutional and national research committee and with the 1964 Helsinki Declaration and its later amendments or comparable ethical standards. The Ethical Committee of the Longgang E.N.T hospital \& Shenzhen Key Laboratory of E.N.T \& Institute of E.N.T Shenzhen Review Board approved the study protocol (2022-0001).

\section{Consent for publication}

Not applicable

\section{Competing interests}

The authors declare that they have no competing interests.

\section{References}


1. K Thomas Robbins, Ashok R Shaha, Jesus E Medina, Joseph A Califano, Gregory T Wolf, Alfio Ferlito, Peter M Som, Terry A Day, Committee for Neck Dissection Classification, American Head and Neck Society. Consensus statement on the classification and terminology of neck dissection. Arch Otolaryngol Head Neck Surg 2008 May;134(5):536-8.

2. Xiangmin Zhang, Folin Liu, Xiaolin Lan, Keqing Luo, Shaojin Li. Combined submandibular gland flap and sternocleidomastoid musculocutaneous flap for postoperative reconstruction in older aged patients with oral cavity and oropharyngeal cancers. World J Surg Oncol. 2014; 12: 259.

3. Bo Gu, Qigen Fang,Yao Wu,Wei Du, Xu Zhang, and Defeng Chen. Impact of submandibular gland preservation in neck management of early-stage buccal squamous cell carcinoma on locoregional control and disease-specific survival. BMC Cancer. 2020; 20: 1034.

4. Shadi Javadi, Bijan Khademi, Mohammad Mohamadianpanah, Mahmoud Shishegar, Amirhossein Babaei. Elective Submandibular Gland Resection in Patients with Squamous Cell Carcinomas of the Tongue. Iran J Otorhinolaryngol. 2021 Jan; 33(114): 23-29.

5. Wei Du, Qigen Fang, Shanting Liu, Defeng Chen, Ruihua Luo, Xu Zhang. Feasibility of Submandibular Gland Preservation in cT1-2N0 Squamous Cell Carcinoma in the Floor of the Mouth. Front Oncol. 2020; 10: 579.

6. Ashish Jakhetiy, MCh , Pallvi Kaul, MS , Arun Pandey, MCh , Tarang Patel, MD , Jitendra Kumar Meena, MD , Mahendra Pal Singh, MCh , Pankaj Kumar Garg, MCh . Distribution and determinants of submandibular gland involvement in oral cavity squamous cell carcinoma. Oral Oncology 118 (2021) 105316.

7. Xiangmin Zhang, Folin Liu, Xiaolin Lan, Lijiang Yu, Wei Wu, Xiuhong Wu, Fufu Xiao, Shaojin Li.Clinical observation of submandibular gland transfer for the prevention of xerostomia after radiotherapy for nasopharyngeal carcinoma: a prospective randomized controlled study of 32 cases.Radiat Oncol. 2014; 9: 62.

8. Ping Zhou, Jing-Xin Chen, Yuan Zhou, Chen-Lu Lian, Bing Yan, San-Gang Wu. Rare Metastasis to the Submandibular Gland in Oral Squamous Cell Carcinoma. Front Oncol. 2021; 11: 728230.

9. Orlando Guntinas-Lichius, Gerd Fabian Volk, Kerry D. Olsen, Antti A. Mäkitie, Carl E. Silver, Mark E. Zafereo, Alessandra Rinaldo, Gregory W. Randolph, Ricard Simo, Ashok R. Shaha, Vincent Vander Poorten, Alfio Ferlito. Facial nerve electrodiagnostics for patients with facial palsy: a clinical practice guideline. Eur Arch Otorhinolaryngol. 2020; 277(7): 1855-1874.

10. Andres Rodriguez-Lorenzo, David Jensson, Wolfgang J. Weninger, Melanie Schmid, Stefan Meng, Chieh-Han John Tzou. Platysma Motor Nerve Transfer for Restoring Marginal Mandibular Nerve Function. Plast Reconstr Surg Glob Open. 2016 Dec; 4(12): e1164.

11. Amr Abdelmonam Abdelaziz M. Elkatatny, Hany Abdelrahim Abdelrazik Abdallah, Dina Ghoraba, Tarek Ahmed Amer, Tarek Hamdy. Hypoglossal Facial Nerve Anastomosis for Post-Operative and Post-Traumatic Complete Facial Nerve Paralysis. Maced J Med Sci. 2019 Dec 15; 7(23): 3984-3996

12. Cheryl L. Currie, Jennifer L. Copeland, M. Lauren Voss, Lisa-Marie Swanepoel, Mirela Ambeskovic, Nimesh B. Patel, Erin K. Higa. Trauma-informed interventions versus control for cancer-risk 
behaviours among adults: rationale and design for a randomized trial. BMC Public Health. 2019; 19 : 1403.

13. Mauro Cozzolino, Stefania Cocco, Michela Piezzo, Giovanna Celia, Susan Costantini, Valentina Abate, Francesca Capone, Daniela Barberio, Laura Girelli, Elisa Cavicchiolo, Paolo Antonio Ascierto, Gabriele Madonna, Alfredo Budillon, Michelino De Laurentiis. A Psychosocial Genomics Pilot Study in Oncology for Verifying Clinical, Inflammatory and Psychological Effects of Mind-Body Transformations-Therapy (MBT-T) in Breast Cancer Patients: Preliminary Results. J Clin Med. 2021 Jan; 10(1): 136.

14. Supasid Jirawatnotai, Pojanan Jomkoh, Tsz Yin Voravitvet, Wuttipong Tirakotai, Natthawut Somboonsap. Computerized Sunnybrook facial grading scale (SBface) application for facial paralysis evaluation. Arch Plast Surg. 2021 May; 48(3): 269-277.

15. Nadimul Hoda, Rajani BC, Subhabrata Ghosh, Sabitha KS, Vasantha Dhara B, Jayesh Nathani.Cervical lymph node metastasis in squamous cell carcinoma of the buccal mucosa: a retrospective study on pattern of involvement and clinical analysis.Med Oral Patol Oral Cir Bucal. 2021 Jan; 26(1): e84-e89.

16. Ding Ding, William Stokes, Megan Eguchi, Mohammad Hararah, Whitney Sumner, Arya Amini, Julie Goddard, Hilary Somerset, Cathy Bradley, Jessica McDermott, David Raben, Sana D. Karam. Association Between Lymph Node Ratio and Recurrence and Survival Outcomes in Patients With Oral Cavity Cancer. JAMA Otolaryngol Head Neck Surg. 2019 Jan; 145(1): 53-61.

17. H. Hakan Coskun, Jesus E. Medina, K. Thomas Robbins, Carl E. Silver, Primož Strojan, Afshin Teymoortash, Phillip K. Pellitteri, Juan P. Rodrigo, Sandro J. Stoeckli, Ashok R. Shaha, Carlos Suçrez, Dana M. Hartl, Remco de Bree, Robert P. Takes, Marc Hamoir, Karen T. Pitman, Alessandra Rinaldo, Alfio Ferlito.Current philosophy in the surgical management of neck metastases for head and neck squamous cell carcinoma.Head Neck. 2015 Jun; 37(6): 915-926.

18. A. Teymoortash, J. A. Werner.Current advances in diagnosis and surgical treatment of lymph node metastasis in head and neck cancer.GMS Curr Top Otorhinolaryngol Head Neck Surg. 2012; 11: Doc04.

19. Shaikh Amjad, Rajiv M. Borle, Mahalaqua Nazli Khatib and Nitin Bhola. Implications of Oral Squamous Cell Carcinoma to Submandibular Gland: A Systematic Analysis. Journal of Pharmaceutical Research International. 2021;33(34A): 144-153.

20. Wen Zeng, Cheng-yu Qiu, Jia-feng Liu, Yiyun Pan, Rong Li, Keqing Luo, Ke-qiang Tian, Fu-fu Xiao, Jun-hui Xie, Xiangmin Zhang.The preservation and application of the submandibular gland in oral squamous cell carcinoma (STROBE) $\square$ Medicine (Baltimore) 2019 Dec; 98(52): e18520.

21. Shlomo A. Koyfman, Nofisat Ismaila, Doug Crook, Anil D'Cruz, Cristina P. Rodriguez, David J. Sher, Damian Silbermins, Erich M. Sturgis, Terance T. Tsue, Jared Weiss, Sue S. Yom, F. Christopher Holsinger.Management of the Neck in Squamous Cell Carcinoma of the Oral Cavity and Oropharynx: ASCO Clinical Practice Guideline.J Clin Oncol. 2019 Jul 10; 37(20): 1753-1774. 
22. Khalid AL-Qahtani, Alex Mlynarek, Jon Adamis, Jeffery Harris, Hadi Seikaly, Tahera Islam. Intraoperative localization of the marginal mandibular nerve: a landmark study.BMC Res Notes. 2015; 8: 382.

23. Khalid AL-Qahtani, Alex Mlynarek, Jon Adamis, Jeffery Harris, Hadi Seikaly, Tahera Islam. Intraoperative localization of the marginal mandibular nerve: a landmark study. BMC Res Notes. 2015; 8: 382.

24. Dimonge Joseph Anthony, Basnayaka Mudiyanselage Oshan Deshanjana Basnayake, Yasith Mathangasinghe, Ajith Peiris Malalasekera. Preserving the marginal mandibular branch of the facial nerve during submandibular region surgery: a cadaveric safety study. Patient Saf Surg. 2018; 12: 23.

25. Vlad Tereshenko, Dominik C. Dotzauer, Udo Maierhofer, Christopher Festin, Matthias Luft, Gregor Laengle, Olga Politikou, Holger J. Klein, Roland Blumer, Oskar C. Aszmann, Konstantin D.

Bergmeister. Selective Denervation of the Facial Dermato-Muscular Complex in the Rat: Experimental Model and Anatomical Basis. Front Neuroanat. 2021; 15: 650761.

26. Paschalis Strantzias, Anna Botou, Arezina Manoli, Panagiotis N Skandalakis, Dimitrios Filippou. Variation of Marginal Mandibular Nerve in a Caucasian Male Cadaver: A Study Using the Anatomage Table. Cureus. 2019 Nov; 11(11): e6168.

27. Ricardo Rodriguez Colon, Jenn J. Park, Daniel Boczar, Gustave K. Diep, Zoe P. Berman, Jorge Trilles, Bachar F. Chaya, Eduardo D. Rodriguez. Evaluating Functional Outcomes in Reanimation Surgery for Chronic Facial Paralysis: A Systematic Review. Plast Reconstr Surg Glob Open. 2021 Mar; 9(3): e3492.

\section{Figures}




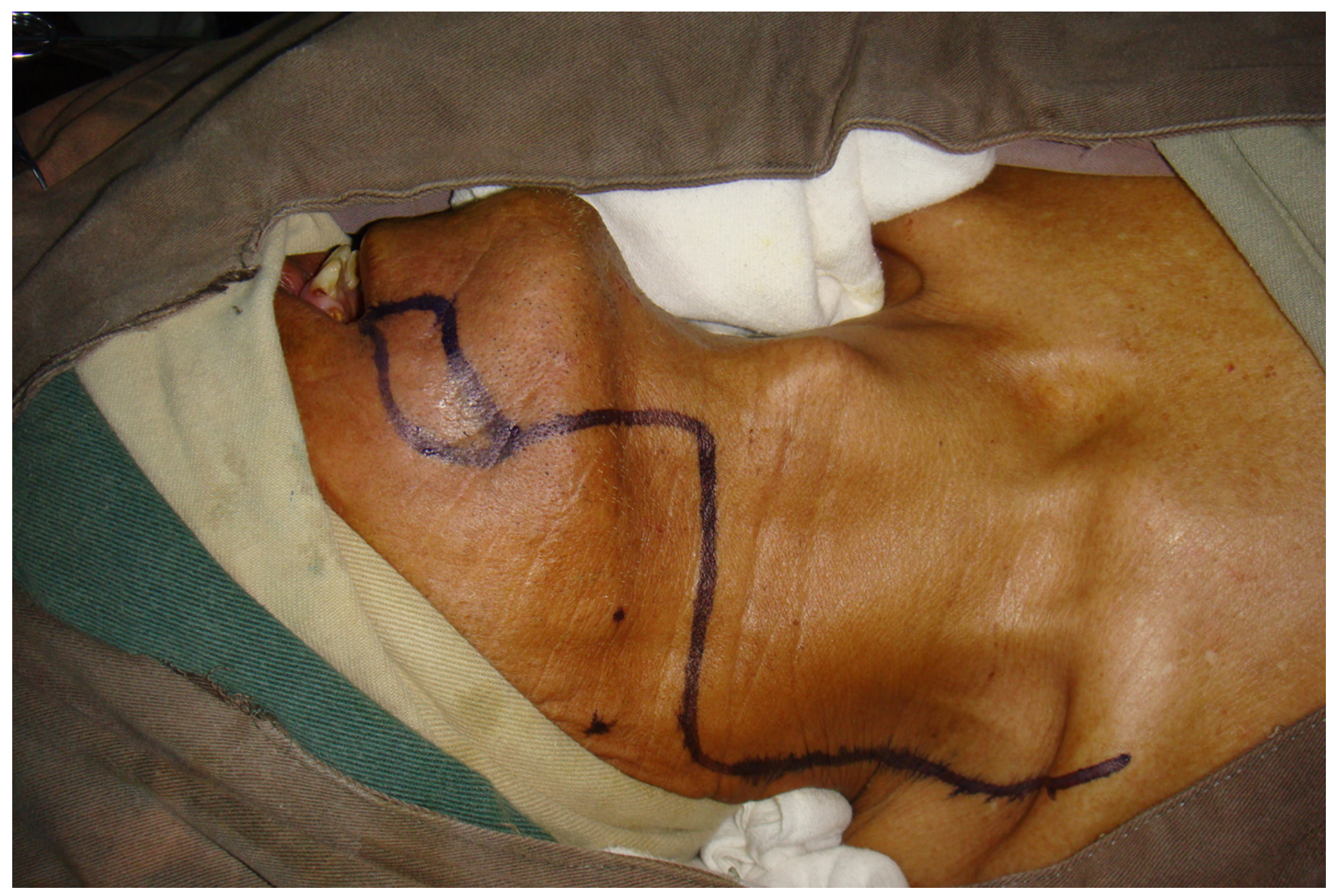

Figure 1

Incision of the buccal mucosa cancer invading skin 


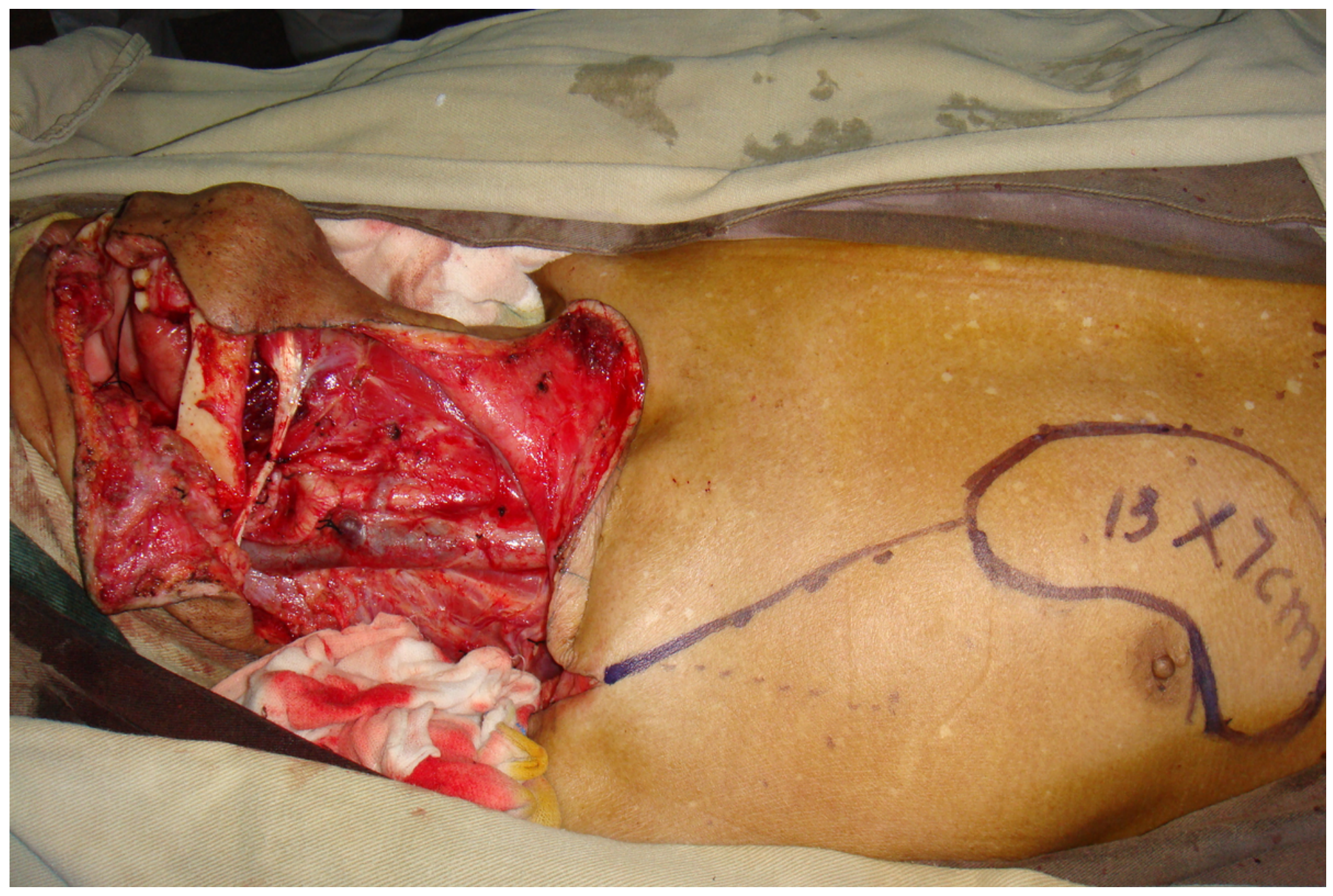

Figure 2

Comprehensive cervical lymphadenectomy with dissection of the marginal mandibular branches of facial nerves plus extended radical resection of the right buccal cancer 


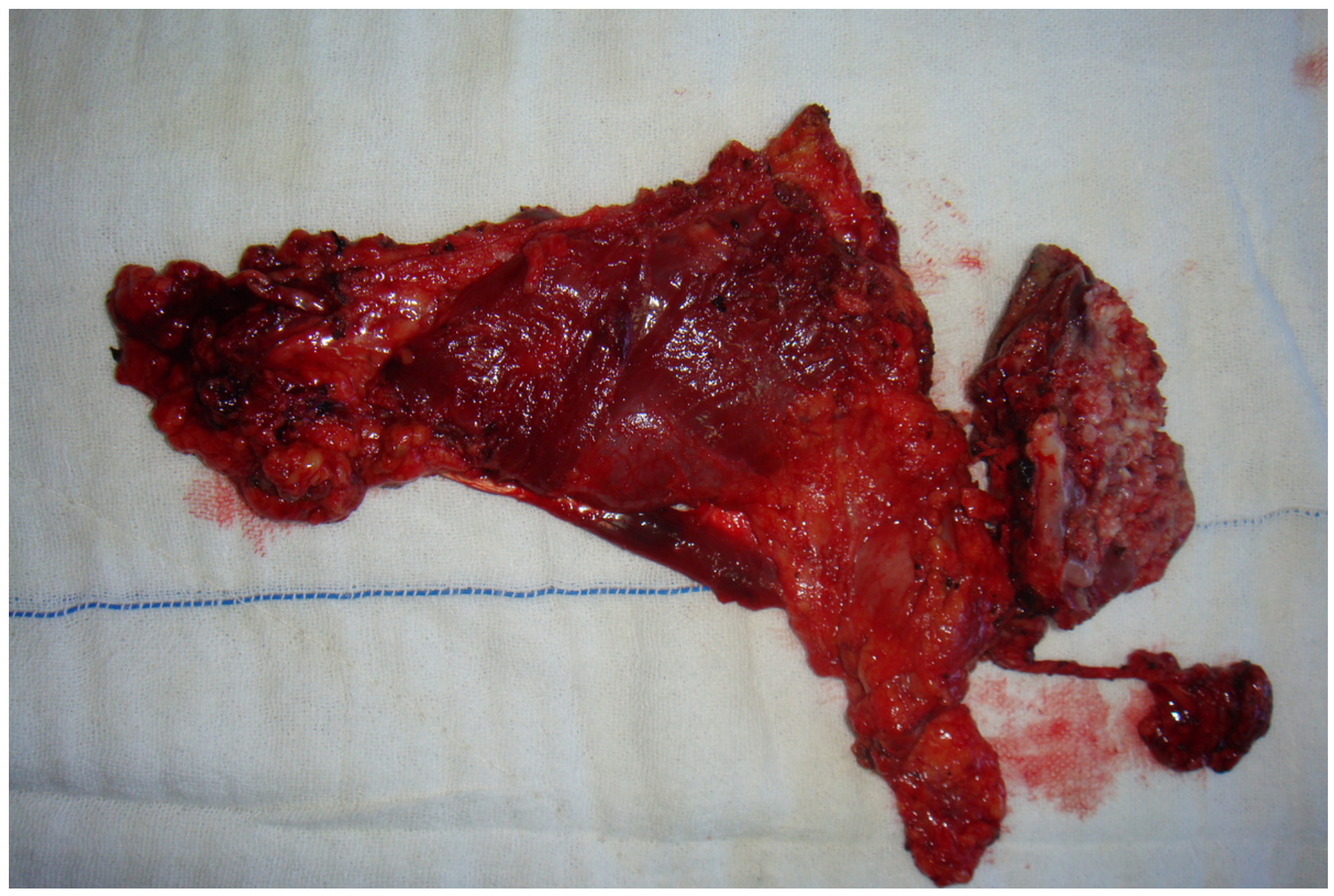

Figure 3

En bloc resection specimen 


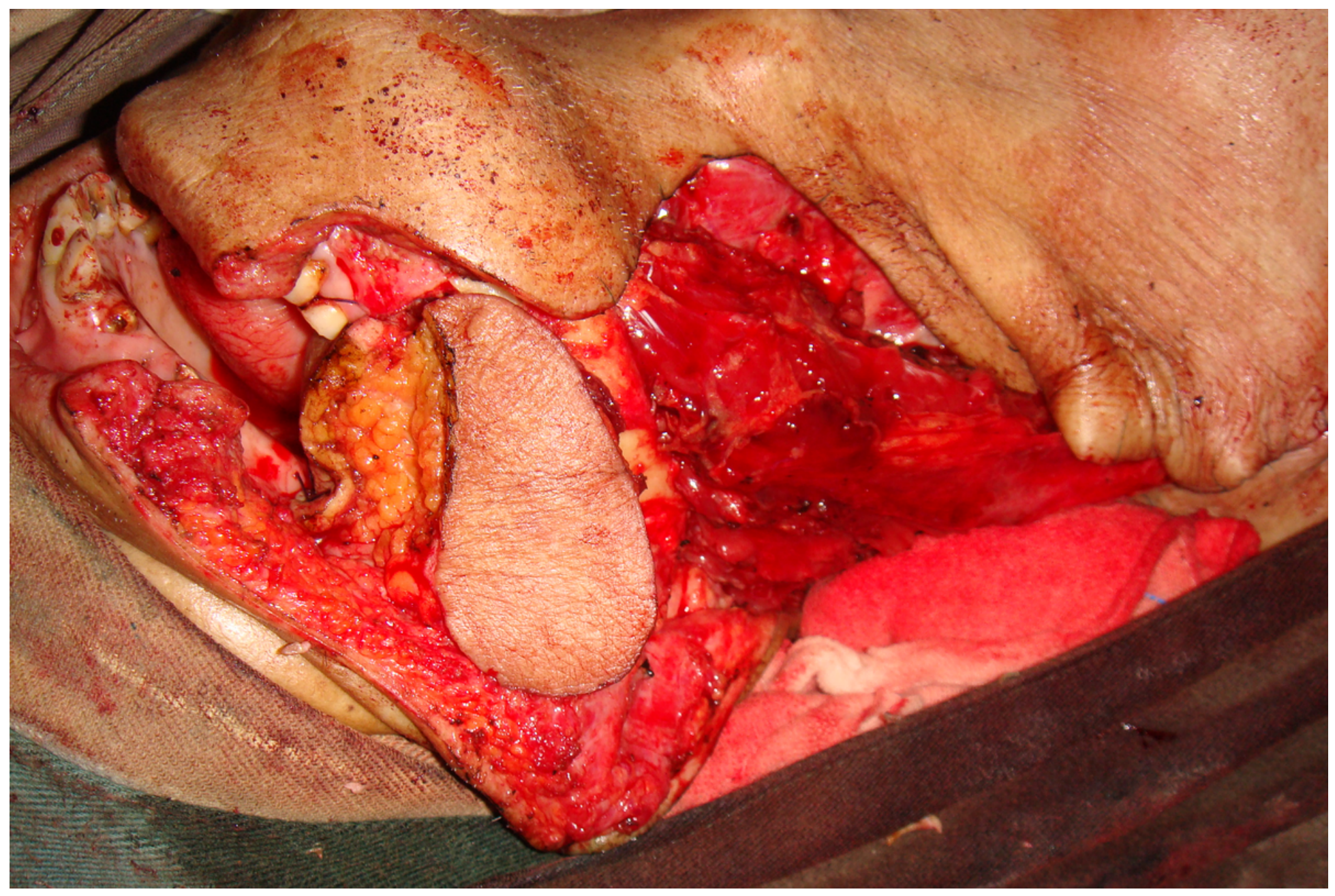

\section{Figure 4}

Repair of penetrating buccal defect with a pectoralis major flap folded in half

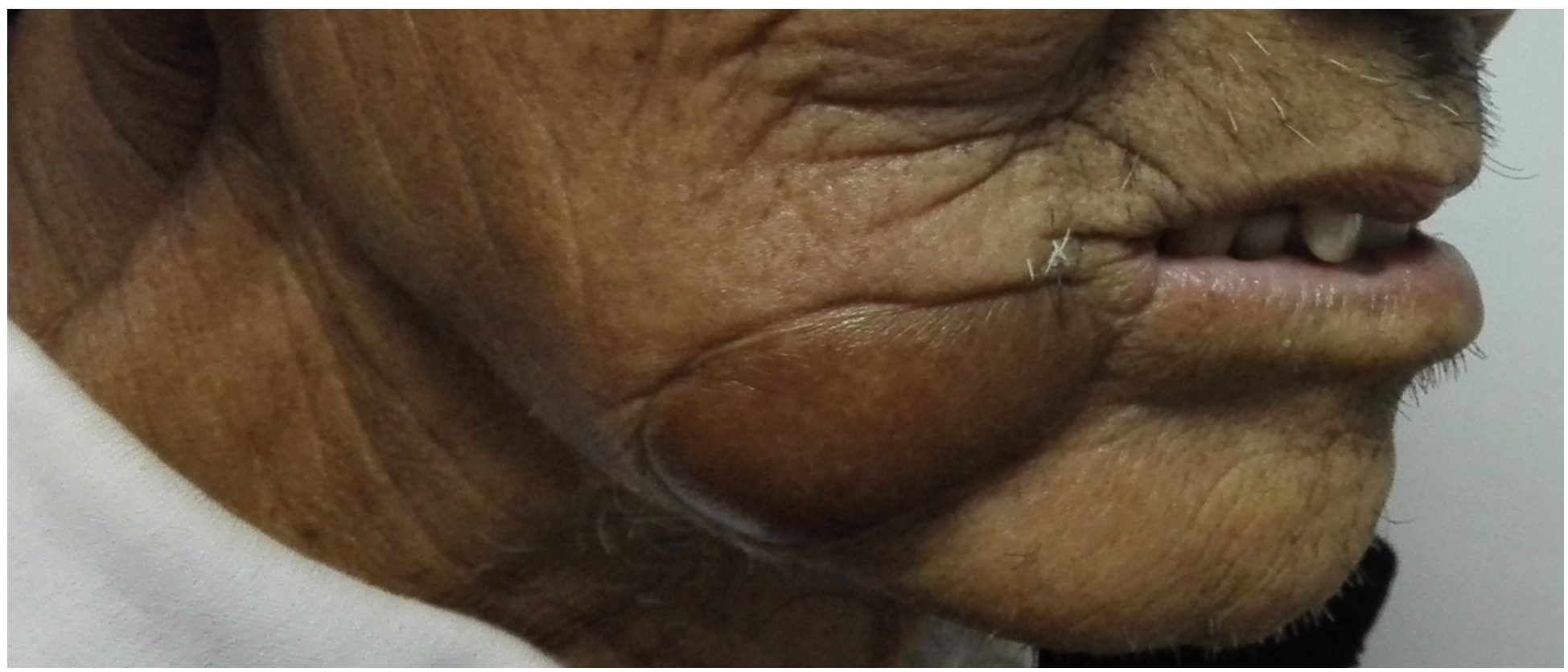

Figure 5 
Re-examination at 3 years after surgery showed that the patient had a good appearance and the marginal mandibular branches of the facial nerves were functioning normally.

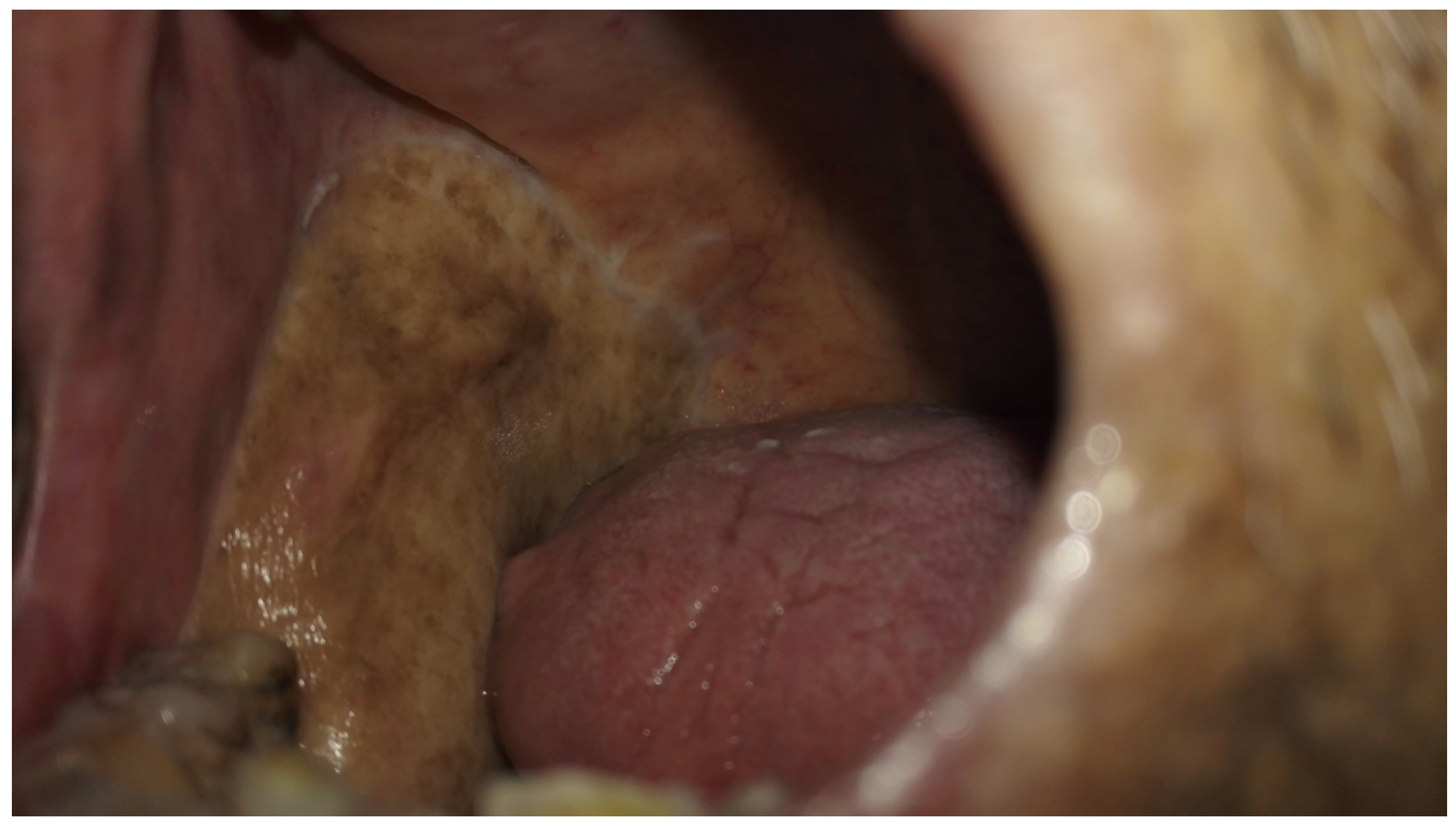

Figure 6

Appearance of the intraoral flap repair 3 years after surgery 


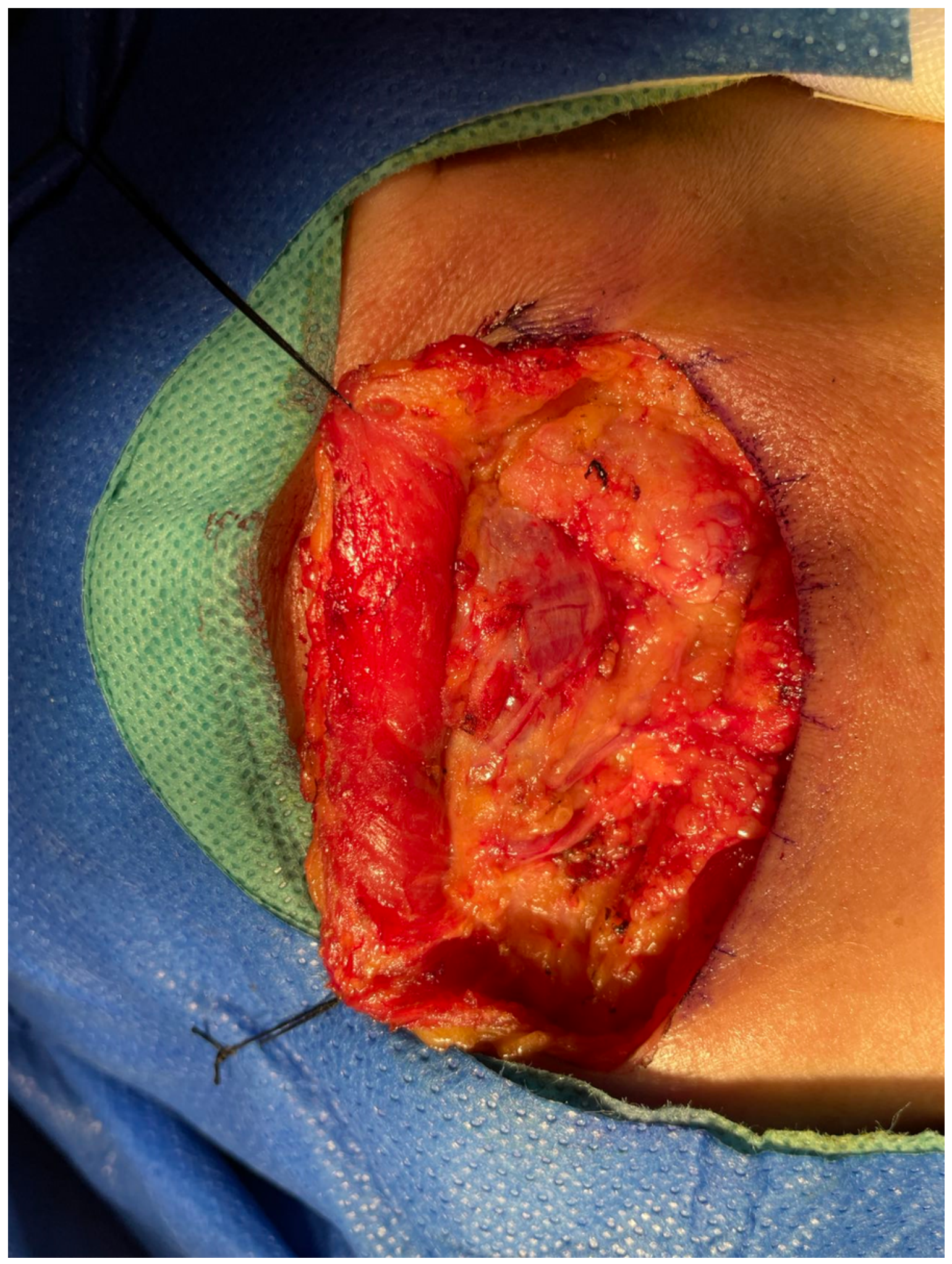

Figure 7

Searching for the marginal mandibular branch of the facial nerve at the mandibular angle 


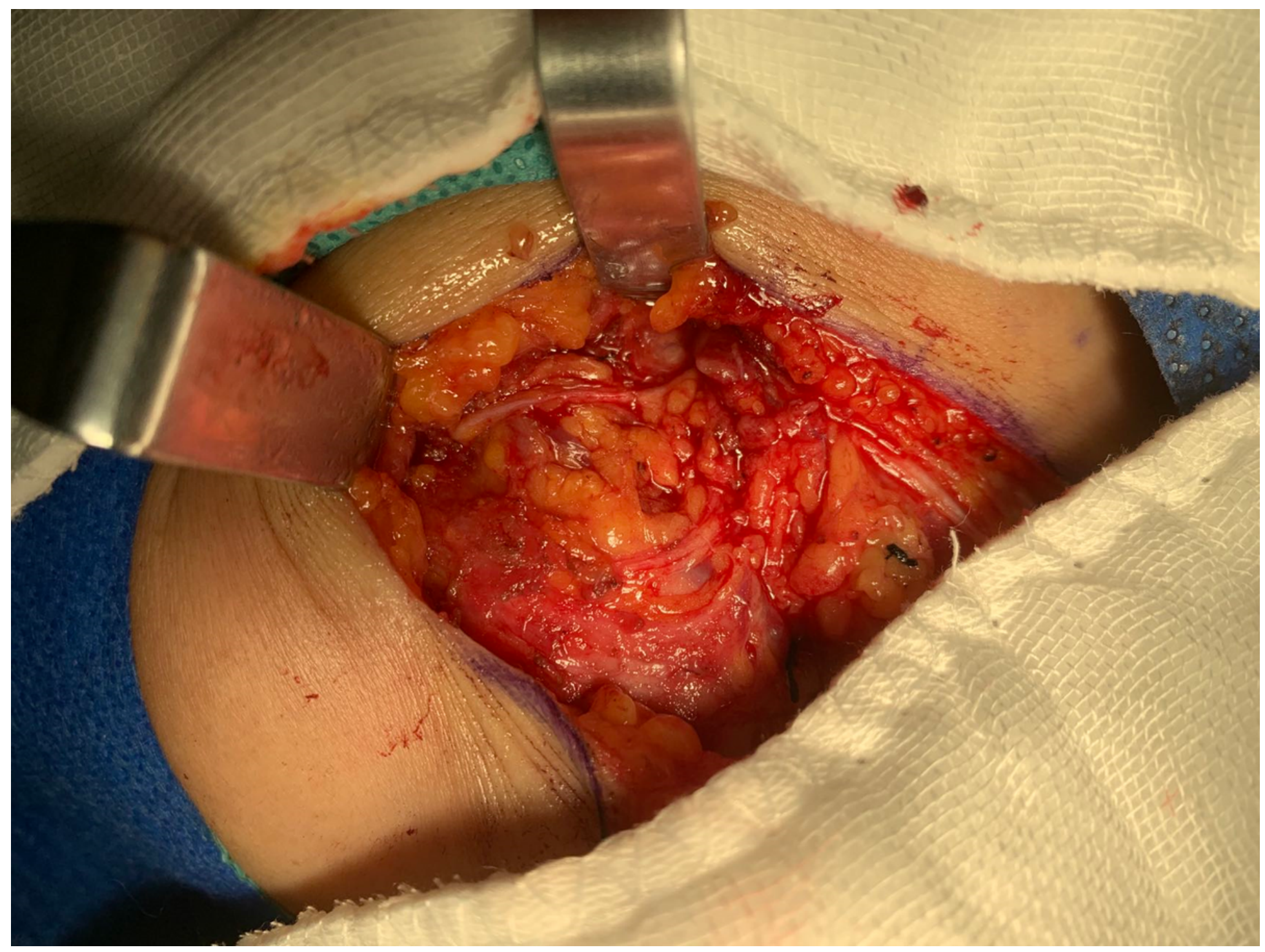

Figure 8

Searching for the marginal mandibular branch of the facial nerve at the distal ends of the facial artery and vein 